\title{
THE ROLE OF N-TERMINAL PRO-BRAIN NATRIURETIC PEPTIDE IN THE ASSESSMENT OF RIGHT VENTRICULAR DYSFUNCTION IN PATIENTS WITH ACUTE PULMONARY EMBOLISM: RESULTS FROM SERBIAN UNIVERSITY PULMONARY EMBOLISM REGISTRY (SUPER)
}

\author{
Ljiljana Kos, Bojan M. Stanetic, Tamara Kovacevic-Preradovic, Miodrag Ostojic, Ljiljana \\ Jovanovic, Vesna Subota, Milena Stavric, Bojana Subotic, Boris Dzudovic, Natasa Novicic, \\ Jovan Matijasevic, Milica Miric, Sonja Salinger, Natasa Markovic-Nikolic, Maja Nikolic, \\ Vladimir Miloradovic, Jelena Marinkovic, Nikola Kocev, Slobodan Obradovic
}

\begin{abstract}
Background: Right ventricular dysfunction (RVD) is a well-known predictor of early death in patients with acute pulmonary embolism and thus early identification of RVD is critical in the risk stratification or management of acute pulmonary embolism (PE).

Aim of this study was to investigate a useful role of cardiac biomarker NTproBNP for predicting echocardiographic right ventricular dysfunction in patients with acute pulmonary embolism.

Methods: A retrospective analysis was performed in 195 consecutive adult patients with pulmonary embolism from the Serbian University Pulmonary Embolism Registry (SUPER 2015-2019) created by six university clinics: Military Medical Academy (Belgrade), Institute of Pulmonary Diseases (Sremska Kamenica), Clinical Center (Nis), University Clinic Zvezdara, Clinical Center (Kragujevac) and University Clinical Centre of Republic of Srpska (Banja Luka). All patients were divided into RVD group and non-RVD group according to whether there was increase in systolic pressure in right ventricle $(>40 \mathrm{mmHg})$ on echocardiography. Odds ratios (OR) and $95 \%$ confidence intervals (CI) assessing the risk factors for RVD were assessed by multivariate logistic regression. The ability of the NT proBNP in predicting the RVD was described by the Receiving Operating Curves analysis.

Results: The mean age of the included patients was $63.9 \pm 1.0$ years, 83/195 (42.6\%) were men, 28/195 (14.4\%) had COPD, 17/195 (8.7\%) had malignancy and 27/195 (13.9\%) were diabetic and 125/195 (64.1\%) had history of arterial hypertension. Patients in the RVD group showed higher right ventricular systolic pressure $(58.89 \pm 15.71$ vs. $35.47 \pm 8.58 ; p<0.001)$, increased right ventricular diameter (33.93 \pm 7.99 vs. $28.38 \pm 3.88 ; p<0.001)$ as well as decreased right ventricular systolic function by tricuspid annular plane systolic excursion (1.66 \pm 0.48 vs. $2.26 \pm 0.29 ; p=0.001)$ when compared with non-RVD group. There was no differences in Troponin I, Troponin $T$ and D-dimer values between the groups $(111.84 \pm 106.51$ vs. $61.38 \pm 76.68 ; p=0.280$, $6.37 \pm 5.63$ vs. $0.64 \pm 0.30 ; p=0.520$, and 6268.71 \pm 9061.35 vs. $4415.47 \pm 4837.94 ; p=0.168$, respectively), whereas $N T$-proBNP was higher in $R V D$ group (6346.56 \pm 7478.27 vs. $2888.45 \pm 4941.94 ; p=0.001)$. In the multivariable logistic regression analysis, NT-proBNP (per $100 \mathrm{pg} / \mathrm{mL}$ ) was independently associated with RVD (OR 1.009 with 95\% CI 1.003$1.015 ; p=0.028)$. In addition, NT-proBNP was found to predict $R V D$ with an area under the curve of 0.711 (95\% CI 0.631-0.791). The cut-off value of NT-proBNP was $704.8 \mu \mathrm{g} / \mathrm{mL}$, with a sensitivity of $81.3 \%$ and specificity of $72.9 \%$.

Conclusion: It appears that NT-proBNP is a strong predictor of echocardiographic RVD in patients with PE. The simple measurements of this cardiac biomarker could be helpful in clinical decision-making or risk stratification in patients with PE.
\end{abstract}

Keywords: Pulmonary embolism; N-terminal pro-brain natriuretic peptide; Right ventricular dysfunction

\section{INTRODUCTION}

Acute pulmonary embolism (PE) is a potentially life-threatening clinical condition with a reported incidence of 70 per 100,000 [1]. PE may be classified into three different types according to hemodynamic conditions in applications. Hemodynamically unstable conditions with hypotension are classified as massive PE. 
Hemodynamically stable conditions with right ventricular dysfunction (RVD) are classified as submassive PE, whereas hemodynamically stable conditions without RVD are classified as nonmassive PE [2]. Massive PE is associated with hemodynamic collapse; it carries a high mortality rate and requires immediate treatment [3]. The appropriate treatment can be started by considering the patient's accompanying comorbid condition. However, additional examinations such as echocardiography are required for the sub-massive PE patient group [4].

Sub-massive PE is regarded as non-massive PE most of the time, which leads to unexpected morbidities and mortalities. This is because submassive PE involves an increase in right ventricular afterload, which leads to right ventricular dilatation and dysfunction. This condition results in right heart failure and eventually death [5-7]. Therefore, investigation of RVD in with non-massive $\mathrm{PE}$ is of high importance.

N-terminal pro-brain natriuretic peptide (NTproBNP) is a member of natriuretic peptides and a useful marker in the evaluation of cardiac functions. It has been shown that NT-proBNP is useful, not only in diagnosis, but also in the risk stratification as a potential predictor of echocardiografic RVD in patients with PE.

In this study, we aimed to investigate the diagnostic value of NT-proBNP for detecting RVD in patients with non-high-risk PE.

\section{Methods}

\section{Study population}

All patients with NT-proBNP from Serbian University Pulmonary Embolism Registry (SUPER 2015-2019) were included in the study. The following clinics were included: Military Medical Academy (Belgrade), Institute of Pulmonary Diseases (Sremska Kamenica), Clinical Center (Nis), University Clinic Zvezdara, Clinical Center (Kragujevac) and University Clinical Centre of Republic of Srpska (Banja Luka). Patients over the age of 18 who presented with symptoms of shortness of breath, hemoptysis, retrosternal pain, or non-specific symptoms and were later diagnosed with PE upon further examinations were included in the study. PE was diagnosed in patients suspected of having PE as a result of laboratory and clinical findings by detection of thrombus in the pulmonary artery and branches with computed tomography pulmonary angiography. Patients with a documented right and left ventricular heart failure before pulmonary embolism, resistant and unregulated hypertension, intracardiac thrombus, sepsis or septic shock, pericardial effusion causing tamponade and serious pleural effusion, nephrotic syndrome, acute kidney and liver failure, serious thyroid hormone dysfunction and other endocrine disorders affecting hemodynamics were excluded from the study.

Demographic characteristics, complaints at the time of presentation, and any comorbid conditions of the patients were recorded in the patient files. Blood samples were taken from patients at the time of presentation for hemogram, biochemistry, and hemostasis testing, and were analysed according to standard procedure. Lower extremity venous Doppler ultrasonography was performed for patients with a positive Homan's test result to detect deep vein thrombosis. Echocardiography was performed for all patients to detect RVD and to evaluate pulmonary artery pressure.

\section{Statistical Analysis}

Categorical variables are presented as numbers and percentages and compared using the chi-square test. Continuous variables are expressed as mean with standard deviation (SD) or median with first and third quartile (Q1-Q3) and compared using a Student $\mathrm{T}$ test or Mann-Whitney $\mathrm{U}$ test test according to the data distribution and number of groups. The Kolmogorov-Smirnov test as well as visual assessment were used to assess the normality of distribution of all continuous variables.

Odds ratios (OR) and 95\% confidence intervals (CI) assessing the risk factors for RVD were assessed by multivariate logistic regression. The following parameters were evaluated in a multivariable model: age, gender, serum creatinine, troponin I, D-dimer and NT proBNP levels. In the analytical methods applied, the level of significance was $\mathrm{p}<0.05$.

The ability of the NT proBNP in predicting the RVD was described by the Receiving Operating Curves analysis. 
All statistical analyses were performed using IBM SPSS Statistics for Macintosh, version 25.0 (IBM, Armonk, New York, USA).

\section{Results}

A total number of 195 patients was included in the study. The mean age of the included patients was $63.9 \pm 1.0$ years, $83 / 195(42.6 \%)$ were men, 28/195 (14.4\%) had COPD, 17/195 (8.7\%) had malignancy and 27/195 (13.9\%) were diabetic and $125 / 195 \quad(64.1 \%)$ had history of arterial hypertension. Baseline characteristics of the study population are listed in Table 1. All patients were divided into RVD group and non-RVD group according to whether there was increased right ventricular systolic pressure $(>40 \mathrm{mmHg})$ on echocardiography. There were no differences in gender, body mass index, chronic obstructive pulmonary disease, heart failure, serum creatinine level, coronary artery disease and type 2 diabetes mellitus between the 2 groups $(\mathrm{p}>0.05)$. As expected, patients in the RVD group showed higher right ventricular systolic pressure $(58.89 \pm 15.71$ vs. $35.47 \pm 8.58 ; \mathrm{p}<0.001)$, increased right ventricular diameter $(33.93 \pm 7.99$ vs. $28.38 \pm 3.88 ; \mathrm{p}<0.001)$ as well as decreased right ventricular systolic function by tricuspid annular plane systolic excursion (1.66 \pm 0.48 vs. $2.26 \pm 0.29 ; \mathrm{p}=0.001)$ when compared with non-RVD group. In addition, patients in RVD group were older $(66.64 \pm 13.01$ vs. $58.78 \pm 16.84$; $\mathrm{p}<0.001)$ and lower $(169.32 \pm 8.39$ vs. $174.45 \pm$ $10.76 ; \mathrm{p}=0.042$ ) than patients in non-RVD group. There was no differences in Troponin I, Troponin T and D-dimer values between the groups $(111.84 \pm$ 106.51 vs. $61.38 \pm 76.68 ; p=0.280,6.37 \pm 5.63$ vs. $0.64 \pm 0.30 ; \mathrm{p}=0.520$, and $6268.71 \pm 9061.35 \mathrm{vs}$. $4415.47 \pm 4837.94 ; \mathrm{p}=0.168$, respectively), whereas NT-proBNP was higher in RVD group (6346.56 \pm 7478.27 vs. $2888.45 \pm 4941.94$; $\mathrm{p}=0.001)$.

In the multivariable logistic regression analysis (Table 2), NT-proBNP (per $100 \mathrm{pg} / \mathrm{mL}$ ) was independently associated with RVD (OR 1.009 with 95\% CI 1.003-1.015; $\mathrm{p}=0.028$ ). Furthermore, the diagnostic odds of independent predictors of RVD were examined with ROC curve analysis (Figure 1). According to the analysis, NT-proBNP was found to predict RVD with an area under the curve of 0.711 (95\% CI 0.631-0.791). The cut-off value of NT-
proBNP was $704.8 \mu \mathrm{g} / \mathrm{mL}$, with a sensitivity of $81.3 \%$ and specificity of $72.9 \%$.

\section{Discussion}

Right ventricular functions take an important place in PE for the evaluation of the clinical condition and determination of the treatment strategy. An acute PE leads to increased right ventricular afterload, right ventricular dilatation and RVD [8]. Presence of RVD in PE is related with poor prognosis. For more than a decade, there has been increased interest in the assessment of the clinical course of PE with cardiac biomarkers. It is known that serum NT-proBNP levels increase after ventricle wall tension. These indicators are used for diagnosis of left ventricular failure related to ventricle dysfunction [9]. In this study, the role of NT-proBNP in the assessment of RVD in PE was evaluated. We found that NT-proBNP levels were significantly higher in patients with RVD compared with patients without RVD. This is in concordance with Kruger et al. [10] who reported that there was a positive relevance between right ventricular enddiastolic pressure and NT-proBNP levels. Similar to our results, Choi et al. [11] reported that there was a positive correlation between NT-proBNP and systolic pulmonary artery pressure in acute PE. Our results showed that serum NT-proBNP levels are increased in acute PE and that this is related to right ventricular functions and systolic pulmonary artery pressure in acute PE. Similar to our results, it was reported that NT-proBNP may be helpful in the assessment of clinical severity in acute PE [12]. We suggest that echocardiography need not be ordinarily ordered in patients with normal NTproBNP levels, because right ventricular function will be almost normal and patients can be considered to be in the low-risk group. If NTproBNP levels are elevated, echocardiography should be performed for further risk stratification and assessment of right ventricular function function.

Measurement of NT-proBNP values can also be helpful for determination of the treatment strategy as well as for prediction of clinical outcome in acute PE. Vuilleumier et al. [13] suggested that the NTproBNP level could be an efficient tool for the identification of very low-risk non-massive PE patients, who could be treated in an outpatient 
setting. It was reported that out-of-hospital treatment is safe in haemodynamically stable patients with $\mathrm{PE}$ with low $(<500 \mathrm{pg} / \mathrm{ml})$ NT-proBNP levels [14]. Binder et al. [15] reported that patients with NTproBNP levels $<1000 \mathrm{pg} / \mathrm{ml}$ could reliably be identified as a low-risk group, as indicated by the high NPV for in-hospital death and the combined primary end-point "complicated clinical course". They suggested a simple risk stratification algorithm for patients with PE, with the use of NT-proBNP testing as an initial step that should be followed by echocardiography in the case of elevated levels of the biomarker. Andreassen et al. [16] reported that NT-proBNP levels may be significant to determine clinical severity and long-term mortality in patients with idiopathic hypertension, chronic pre-capillary hypertension and PE. In our study, we did not evaluate the patients with respect to clinical outcome.

\section{Study limitations}

The study has several limitations. The study is limited by the small sample size, which precludes the possibility of any strong conclusions. Prospective and controlled studies involving larger numbers of acute PE patients are needed. In addition, only one blood sample was collected from each patient on admission for measuring NTproBNP levels. Since, this is a retrospective analysis, selection bias can not be excluded.

\section{Conclusion}

It appears that NT-proBNP levels are associated with RVD and can be used as a marker for the presence of RVD in patients with acute PE. It has been shown that NT-proBNP has good diagnostic value for RVD in patients with non-high-risk PE, but a clinical application needs a comprehensive evaluation combined with the overall manifestations of the disease.

\section{References}

1. Silverstein MD, Heit JA, Mohr DN. Trends in the incidence of deep vein thrombosis and pulmonary embolism: a 25-year population-based study. Arch Intern Med 1998; 158(6):585-593

2. Cho JH, Kutti Sridharan G, Kim SH. Right ventricular dysfunction as an echocardiographic prognostic factor in hemodynamically stable patients with acute pulmonary embolism: a meta- analysis. BMC Cardiovasc Disord 2014; 14:64

3. Kucher N, Rossi E, De Rosa M, Goldhaber SZ. Massive pulmonary embolism. Circulation 2006; 113(4):577-582

4. Alpert JS, Smith R, Carlson J. Mortality in patients treated for pulmonary embolism. JAMA 1976; 236(13):1477-1480

5. Grifoni S, Olivotto I, Cecchini P. Short- term clinical outcome of patients with acute pulmonary embolism, normal blood pressure, and echocardiographic right ventricular dysfunction. Circulation 2000; 101(24):2817-2822

6. Meyer T, Binder L, Hruska N. Cardiac troponin I elevation in acute pulmonary embolism is associated with right ventricular dysfunction. J Am Coll Cardiol 2000; 36(5):1632-1636

7. Pieralli F, Olivotto I, Vanni S. Usefulness of bedside testing for brain natriuretic peptide to identify right ventricular dysfunction and outcome in normotensive patients with acute pulmonary embolism. Am J Cardiol 2006; 97(9):1386-1390

8. Torbicki A, Perrier A, Konstantinides S, Agnelli G, Galiè N, Pruszczyk P. Guidelines on the diagnosis and management of acute pulmonary embolism: the Task Force for the Diagnosis and Management of Acute Pulmonary Embolism of the European Society of Cardiology. Eur Heart J 2008; 29: 2276-315.

9. Ruskoaho H. Cardiac hormones as diagnostic tools in heart failure. Endocr Rev 2003; 24: 341-56.

10. Krüger S, Graf J, Merx MW, Koch KC, Kunz D, Hanrath P. Brain natriuretic peptide predicts right heart failure in patients with acute pulmonary embolism. Am Heart J 2004; 147: 60-5.

11. Choi HS, Kim KH, Yoon HJ, Hong YJ, Kim JH, Ahn Y. Usefulness of cardiac biomarkers in the prediction of right ventricular dysfunction before echocardiography in acute pulmonary embolism. J Cardiol 2012; 60: 508-13.

12. Pruszczyk P, Kostrubiec M, Bochowicz A, Styczy?ski G, Szulc M, Kurzyna M. N-terminal probrain natriuretic peptide in patients with acute pulmonary embolism. Eur Respir J 2003; 22: 649-53.

13. Vuilleumier N, Le Gal G, Cornily JC, Hochstrasser D, Bounameaux H, Aujesky D. Is NT-proBNP superior to clinical scores for risk stratification in non-massive pulmonary embolism? J Thromb Haemost 2010; 8: 1433-5.

14. Agterof MJ, Schutgens RE, Snijder RJ, Epping G, Peltenburg HG, Posthuma EF. Out of hospital treatment of acute pulmonary embolism in patients 
with a low NT-proBNP level. J Thromb Haemost 2010; 8: 1235-41.

15. Binder L, Pieske B, Olschewski M, Geibel A, Klostermann B, Reiner C. N-terminalpro-brain natriuretic peptide or troponin testing followed by echocardiography for risk stratification of acutepulmonary embolism. Circulation 2005; 112: 1573-9.

16. Andreassen AK, Wergeland R, Simonsen S, Geiran $\mathrm{O}$, Guevara C, Ueland T. N-terminal pro-B-type natriuretic peptide as an indicator of disease severity in a heterogeneous group of patients with chronic precapillary pulmonary hypertension. Am J Cardiol 2006; 98: 525-9.

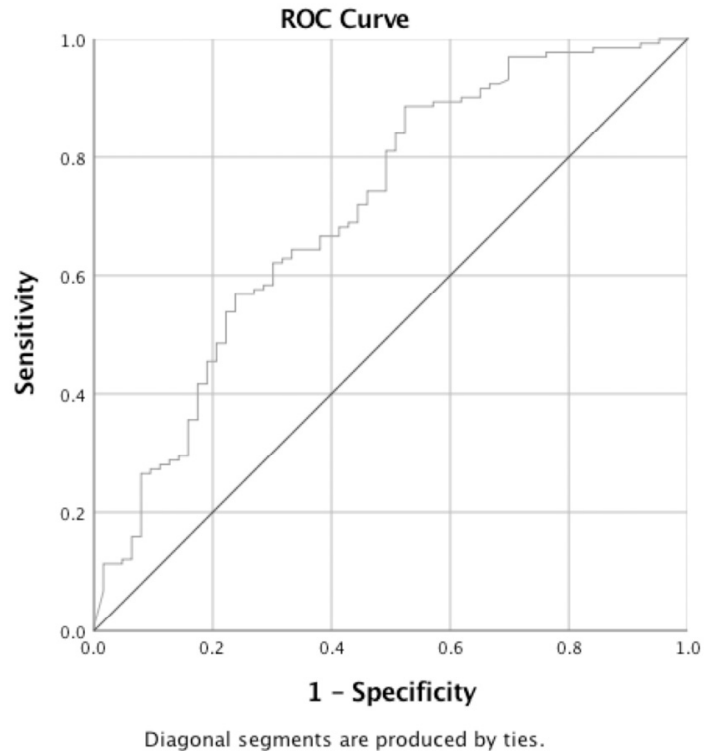

Diagonal segments are produced by ties.

Table 1. Baseline characteristics.

\begin{tabular}{|c|c|c|c|c|}
\hline \multicolumn{4}{|c|}{ RV dysfunction (RV systolic pressure $>40 \mathrm{mmHg}$ ) } & \multirow{3}{*}{$\begin{array}{l}\text { metrics } \\
\text { years }\end{array}$} \\
\hline & $\begin{array}{l}\text { RVD } \\
n=132\end{array}$ & $\begin{array}{c}\text { Non-RVD } \\
n=63\end{array}$ & $\mathrm{P}$ & \\
\hline Age & $66.64 \pm 13.01$ & $58.78 \pm 16.84$ & $<0.001$ & \\
\hline Male & $53(40.2 \%)$ & $30(47.6 \%)$ & 0.355 & \\
\hline Body weight & $74.81 \pm 13.92$ & $82.14 \pm 21.99$ & 0.090 & $\mathrm{~kg}$ \\
\hline Body height & $169.32 \pm 8.39$ & $174.45 \pm 10.76$ & 0.042 & $\mathrm{~cm}$ \\
\hline BMI & $26.54 \pm 4.92$ & $27.52 \pm 5.32$ & 0.466 & \\
\hline RVsystolic pressure & $58.89 \pm 15.71$ & $35.47 \pm 8.58$ & $<0.001$ & $\mathrm{mmHg}$ \\
\hline RVd (PLAX) & $33.93 \pm 7.99$ & $28.38 \pm 3.88$ & $<\mathbf{0 . 0 0 1}$ & $\mathrm{mm}$ \\
\hline TAPSE & $1.66 \pm 0.48$ & $2.26 \pm 0.29$ & 0.001 & $\mathrm{~mm}$ \\
\hline Pacc & $62.53 \pm 22.15$ & $98.63 \pm 42.66$ & 0.010 & msec \\
\hline Troponin $\mathrm{T}$ & $111.84 \pm 106.51$ & $61.38 \pm 76.68$ & 0.280 & $\mu \mathrm{mol} / 1$ \\
\hline Troponin I & $6.37 \pm 5.63$ & $0.64 \pm 0.30$ & 0.520 & $\mathrm{mmol} / \mathrm{l}$ \\
\hline D-Dimer & $6268.71 \pm 9061.35$ & $4415.47 \pm 4837.94$ & 0.168 & \\
\hline NT-proBNP & $6346.56 \pm 7478.27$ & $2888.45 \pm 4941.94$ & 0.001 & $\mathrm{pg} / \mathrm{mL}$ \\
\hline Glucose & $8.94 \pm 5.12$ & $7.33 \pm 4.60$ & 0.041 & $\mathrm{mmol} / \mathrm{L}$ \\
\hline Serum creatinine & $109.04 \pm 55.82$ & $100.56 \pm 68.61$ & 0.360 & $\mu \mathrm{mol} / \mathrm{L}$ \\
\hline Hemoglobine & $133.10 \pm 21.98$ & $129.92 \pm 22.45$ & 0.355 & $\mathrm{~g} / \mathrm{L}$ \\
\hline Hematocrite & $0.39 \pm 0.05$ & $0.39 \pm 0.05$ & 0.459 & $\mathrm{~L} / \mathrm{L}$ \\
\hline COPD & $20(15.2 \%)$ & $8(12.7 \%)$ & 0.648 & \\
\hline LVEF & $57.18 \pm 10.32$ & $57.22 \pm 9.59$ & 0.983 & $\%$ \\
\hline Spontaneous PE & $78(60.0 \%)$ & $34(54.0 \%)$ & 0.441 & \\
\hline Acute PE & $127(96.9 \%)$ & $59(93.7 \%)$ & 0.411 & \\
\hline Malignancy & $11(8.4 \%)$ & $6(9.5 \%)$ & 0.785 & \\
\hline DVT & $30(23.6 \%)$ & $19(31.7 \%)$ & 0.243 & \\
\hline Arterial hypertension & $94(71.2 \%)$ & $31(49.2 \%)$ & 0.003 & \\
\hline Coronary artery disease & $15(11.4 \%)$ & $8(12.7 \%)$ & 0.787 & \\
\hline Type 2 diabetes mellitus & $18(13.6 \%)$ & $9(14.3 \%)$ & 0.902 & \\
\hline
\end{tabular}

Data given as mean \pm standard deviation or $\mathrm{n}(\%)$. 
Table 2. Multivariate logistic regression analysis showing associations between the different factors and the right

\begin{tabular}{lllll}
\hline \multicolumn{5}{c}{ venctricular dysfunction at admission. } \\
\hline Variables & B & OR & $\mathbf{9 5 \%}$ CI & p-value \\
\hline Age & -0.044 & 0.957 & $0.920-0.995$ & 0.026 \\
Serum creatinine & 0.005 & 1.005 & $0.996-1.014$ & 0.300 \\
Male & -0.158 & 0.854 & $0.301-2.423$ & 0.766 \\
Troponin I & -0.066 & 0.936 & $0.766-1.144$ & 0.517 \\
D-dimere & 0.001 & 1.003 & $0.945-1.184$ & 0.816 \\
NT proBNP & 0.000 & 1.009 & $1.003-1.015$ & 0.028 \\
\hline
\end{tabular}

Ljiljana Kos

Department of Cardiology, University Clinical Centre of the Republic of, Banja Luka, Bosnia and Herzegovina Corresponding author at e-mail address: ljiljana.kos@kc-bl.com (Lj.Kos). 